\title{
Dual CCR2/CCR5 antagonist treatment attenuates adipose inflammation, but not microvascular complications in ob/ ob mice
}

\author{
Phillipe D. O'Brien PhD ${ }^{1}$ | Lucy M. Hinder $\mathrm{PhD}^{1}$ | Sebastian D. Parlee $\mathrm{PhD}^{2}$ | \\ John M. Hayes BA ${ }^{1}$ | Carey Backus BA ${ }^{1}$ | Hongyu Zhang MD, MSC $^{3}$ | Lijun Ma PhD ${ }^{4}$ | \\ Stacey A. Sakowski ${ }^{5}$ | Frank C. Brosius III MD ${ }^{3}$ | Eva L. Feldman MD, PhD ${ }^{1}$ ๑
}

${ }^{1}$ Department of Neurology, University of Michigan, Ann Arbor, Michigan

${ }^{2}$ Department of Molecular \& Integrative Physiology, University of Michigan, Ann Arbor, Michigan

${ }^{3}$ Division of Nephrology, Department of Internal Medicine, University of Michigan, Ann Arbor, Michigan

${ }^{4}$ Department of Cardiometabolic Disease, Merck Research Laboratories, Kenilworth, New Jersey

${ }^{5}$ A. Alfred Taubman Medical Research Institute, University of Michigan, Ann Arbor, Michigan

\section{Correspondence}

Eva L. Feldman MD, PhD, Russell N. DeJong Professor of Neurology, 5017 AATBSRB, 109 Zina Pitcher Place, Ann Arbor, Michigan 48109.

Email: efeldman@umich.edu

Funding information

Funding was provided by the National Institutes of Health (1DP3DK094292, 1R24082841 to

E. L. F and F. C. B.); Novo Nordisk Foundation (NNF14SA0006 to E.L.F.), Juvenile Diabetes Research Foundation (Post-doctoral Fellowship to L. M. H.), Milstein, Nathan and Rose Research Fund; Sinai Medical Staff Foundation

Neuroscience Scholar Fund 2; Robert C Graham Fund; Walbridge Aldinger Graduate Fellowship Fund (Post-doctoral Fellowship to P. D. O); American Diabetes Association; Program for Neurology Research and Discovery; A. Alfred Taubman Medical Research Institute; and the University of Michigan Center for Organogenesis Non-Traditional Post-Doctoral Fellowship (to S. D. P).
Diabetic peripheral neuropathy (DPN) and diabetic kidney disease (DKD) are common diabetic complications with limited treatment options. Experimental studies show that targeting inflammation using chemokine receptor (CCR) antagonists ameliorates DKD, presumably by reducing macrophage accumulation or activation. As inflammation is implicated in DPN development, we assessed whether CCR2 and CCR5 antagonism could also benefit DPN. Five-week-old ob/ ob mice were fed a diet containing MK-0812, a dual CCR2-CCR5 receptor antagonist, for 8 weeks; DPN, DKD and metabolic phenotyping were then performed to determine the effect of CCR inhibition. Although MK-0812 reduced macrophage accumulation in adipose tissue, the treatment had largely no effect on metabolic parameters, nerve function or kidney disease in ob/ob mice. These results conflict with published data that demonstrate a benefit of CCR antagonists for DKD and hyperglycaemia. We conclude that CCR signaling blockade is ineffective in ob/ob mice and suspect that this is explained by the severe hyperglycaemia found in this model. It remains to be determined whether MK-0812 treatment, alone or in combination with improved glycaemic control, is useful in preventing diabetic complications in alternate animal models.

\section{KEYWORDS}

animal pharmacology, diabetes complications, diabetic nephropathy, diabetic neuropathy, drug mechanism, mouse model

\section{1 | INTRODUCTION}

Systemic inflammation, primarily driven by adipose tissue dysfunction, is recognized as an underlying contributor to the development of type 2 diabetes (T2D). ${ }^{1}$ Inflammation is also implicated in diabetic kidney disease (DKD) and diabetic peripheral neuropathy (DPN), 2 common microvascular complications of T2D., ${ }^{2,3}$ As T2D patients manifest with hyperglycaemia, current prevention strategies focus on 
glycaemic control; however, this approach does not guarantee protection from DKD or DPN. ${ }^{4,5}$ Thus, targeting inflammation may provide an adjuvant therapy for the prevention of diabetic complications.

A hallmark of DKD is leukocyte accumulation, including macrophages, within the kidney glomerulus where they secrete proinflammatory proteins such as tumor necrosis factor alpha (TNF $\alpha$ ), promoting inflammation and subsequent kidney injury. ${ }^{3}$ Indeed, macrophage accumulation strongly correlates with renal dysfunction in patients with DKD. ${ }^{6}$ Although not as well characterized, studies support a similar inflammatory mechanism in DPN. ${ }^{7,8}$

During initial tissue injury, resident leukocytes become activated and secrete chemokines that bind to chemokine receptors (CCRs) found on circulating leukocytes. This chemokine-CCR signaling facilitates trafficking of circulating leukocytes to the site of injury where they propagate inflammation. Unlike inflammation triggered by external pathogens, metabolic inflammation is difficult to resolve, leading to low-grade, chronic activation of the immune response. ${ }^{1}$ Sustained inflammation promotes leukocyte accumulation in the damaged tissue, the continued production of pro-inflammatory mediators, and
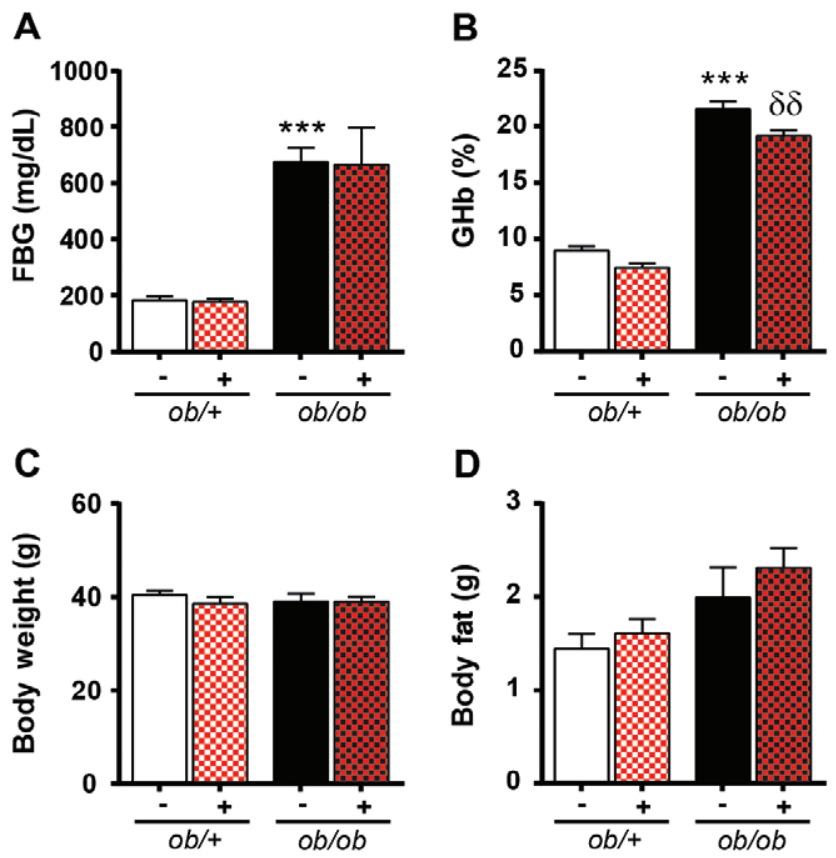

D
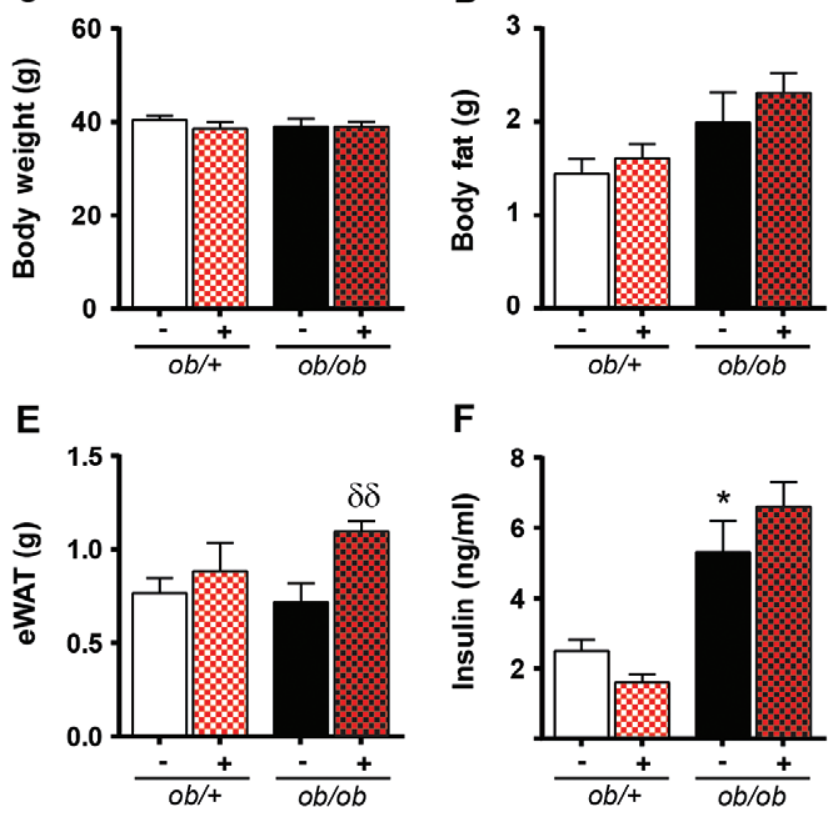

$\mathbf{F}$

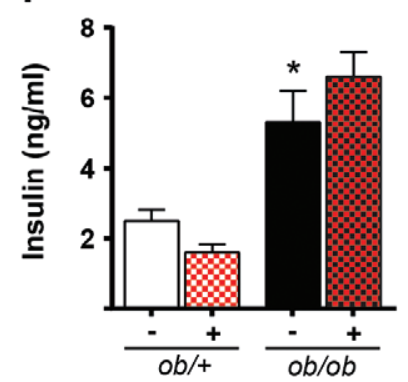

amplification of the inflammatory response via a positive feedback loop. Chemokine C-C ligand-2 (CCL2) is responsible for recruiting CCR2-expressing leukocytes, including macrophages, to sites of inflammation. Antagonizing CCL2 signaling attenuates features of T2D in mouse models by preventing leukocyte infiltration into adipose tissue. ${ }^{9}$ Importantly, these benefits appear to extend to diabetic complications, as CCR2 antagonism reduces renal tissue inflammation and ameliorates DKD in mouse models of T2D. ${ }^{10}$ Likewise, in patients with DKD, treatment with a CCR2 antagonist or an inhibitor of CCL2/MCP1 can reduce proteinuria. ${ }^{11}$ Though less well studied, CCL5-CCR5-mediated signaling has also been implicated in T2D patients and rodent models of $\mathrm{DKD}^{12}$; however, unlike DKD, CCR2 and CCR5 activation in DPN has not yet been examined.

We recently completed microarray gene expression analyses and reported that biological pathways related to inflammation, including chemotaxis, immune response and response to wounding, are dysregulated in the sciatic nerve of $o b / o b$ and $d b / d b$ mice. ${ }^{13}$ Moreover, the expression levels of chemokines $\mathrm{Cl} 2, \mathrm{Cll} 4$ and $\mathrm{C} / 8$, ligands for CCR2 and CCR5, were increased in sciatic nerve tissue of ob/ob mice. These data suggest that, similar to DKD, chemokine-CCR signaling is involved in DPN. Therefore, we tested whether blocking CCR2- and CCR5mediated chemokine signaling would prevent DPN as well as DKD in ob/ob mice, a mouse model of both microvascular complications. ${ }^{13,14}$

\section{RESEARCH DESIGN AND METHODS}

\subsection{Animals and diets}

Male $o b /+$ and $o b / o b$ mice (BTBR.Cg-Lepob/WiscJ) were fed a standard diet $(11.5 \% \mathrm{kcal}$ fat) or a diet containing MK-0812 $(10 \mathrm{mg} / \mathrm{kg} /$ mouse) ad libitum from 5 to 13 weeks of age. Fasting blood glucose (FBG), percent glycated haemoglobin (\%GHb) and plasma insulin were measured at study conclusion. Additional details into experimental procedures are described in Appendix S1, Supporting Information. Details on the pharmacokinetic profile (Table S2) and primer sequences for gene expression analysis (Table S3) are provided in Supporting Information.

\section{2 | DPN and DKD phenotyping}

DPN and DKD phenotyping was performed on the same animals in accordance with Diabetic Complications Consortium guidelines (http://www.diacomp.org) and is described in Appendix S1, Supporting Information. Briefly, DPN phenotyping consisted of assessing hindpaw withdrawal from a thermal stimulus, sensory and motor nerve conduction velocities (NCV), and intraepidermal nerve fiber (IENF) density; DKD phenotyping consisted of measuring urinary albumin excretion (UAE), the albumin creatinine ratio (ACR), and the mesangial index quantified by calculating the percentage of the total glomerular tuft area that was PAS-positive.

\section{3 | Statistical analysis}

Statistical analysis consisted of one-way ANOVA with Tukey's posttest for multiple comparisons or Kruskal-Wallis test with Dunn's 
post-test for multiple comparisons, as appropriate, using GraphPad Prism Software, Version 6 (GraphPad Software, La Jolla, California). Error bars represent SEM ( $n=6$ mice/group). A $P$ value of $<.05$ was considered statistically significant.

\section{3 | RESULTS}

\section{1 | MK-0812 treatment does not affect FBG, \% $\mathrm{GHb}$, insulin or body weight}

At 13 weeks, ob/ob mice had increased FBG, \%GHb and insulin when compared to non-diabetic controls (Figure 1A,B,F). Body weight was similar in $o b /+$ and ob/ob mice (Figure $1 C$ ); however, the increase in total adipose tissue mass in ob/ob mice (Figure 1D) suggests that $o b /$ + mice have greater lean muscle mass. MK-0812 had no effect on FBG, weight, total adipose mass or insulin levels in $o b /+$ or $o b / o b$ mice (Figure 1A,C,D,F) and only a modest effect on \%GHb (Figure 1B). Moreover, MK-0812 treatment increased eWAT mass in $o b / o b$ mice (Figure 1E).

\section{2 | MK-0812 treatment does not prevent development of diabetic microvascular complications}

DPN and DKD measures were examined in ob/ob mice to determine the effects of MK-0812 on diabetic complications. ob/ob mice developed DPN, with increased hindpaw withdrawal latency (Figure 2A), reduced IENF (Figure $2 \mathrm{~B}$ ) and reduced sensory (Figure $2 \mathrm{C}$ ) and motor (Figure 2D) NCV compared to $o b /+$ mice. ob/ob mice also exhibited features of DKD, with increased ACR (Figure 2E), polyuria (Figure 2F), mesangial matrix expansion (Figure 2G) and glomerular thickening (Figure 2H). MK-0812-treated ob/ob mice showed no improvement in diabetic nerve (Figure 2A-D) or kidney changes (Figure $2 \mathrm{E}-\mathrm{H}$ ) relative to untreated controls, indicating that $\mathrm{MK}-0812$ treatment did not prevent DPN or DKD.

\section{3 | Effects of MK-0812 treatment on systemic, neuronal, renal and adipose inflammation}

To determine the effects of MK-0812 treatment on systemic and local inflammation, a cytokine/chemokine multiplex array was performed on plasma and nerve (SCN/DRG) and kidney (glomerulus) tissue lysates. Interestingly, little to no difference in inflammatory protein levels was observed between ob/ob mice and ob/+ mice in plasma or tissue (Table S1, Supporting Information). The multiplex data were validated by more sensitive ELISAs for plasma TNF- $\alpha$, IL6 and CCL2 (Figure S1, Supporting Information). As adipose tissue inflammation drives insulin resistance and is implicated in T2D development, ${ }^{1}$ changes in markers of macrophage inflammation were also assessed in eWAT. ob/ob mice had increased mRNA expression of inflammation-related mediators, including $\mathrm{Cd} 68$, F4/80, Pai-1 and Tnf $\alpha$ (Figure 2A-D), while MK-0812 treatment decreased expression of Cd68, F4/80 and Tnf $\alpha$ (Figure S2, Supporting Information). These data suggest that MK-0812 effectively suppressed macrophage infiltration in ob/ob adipose tissue.
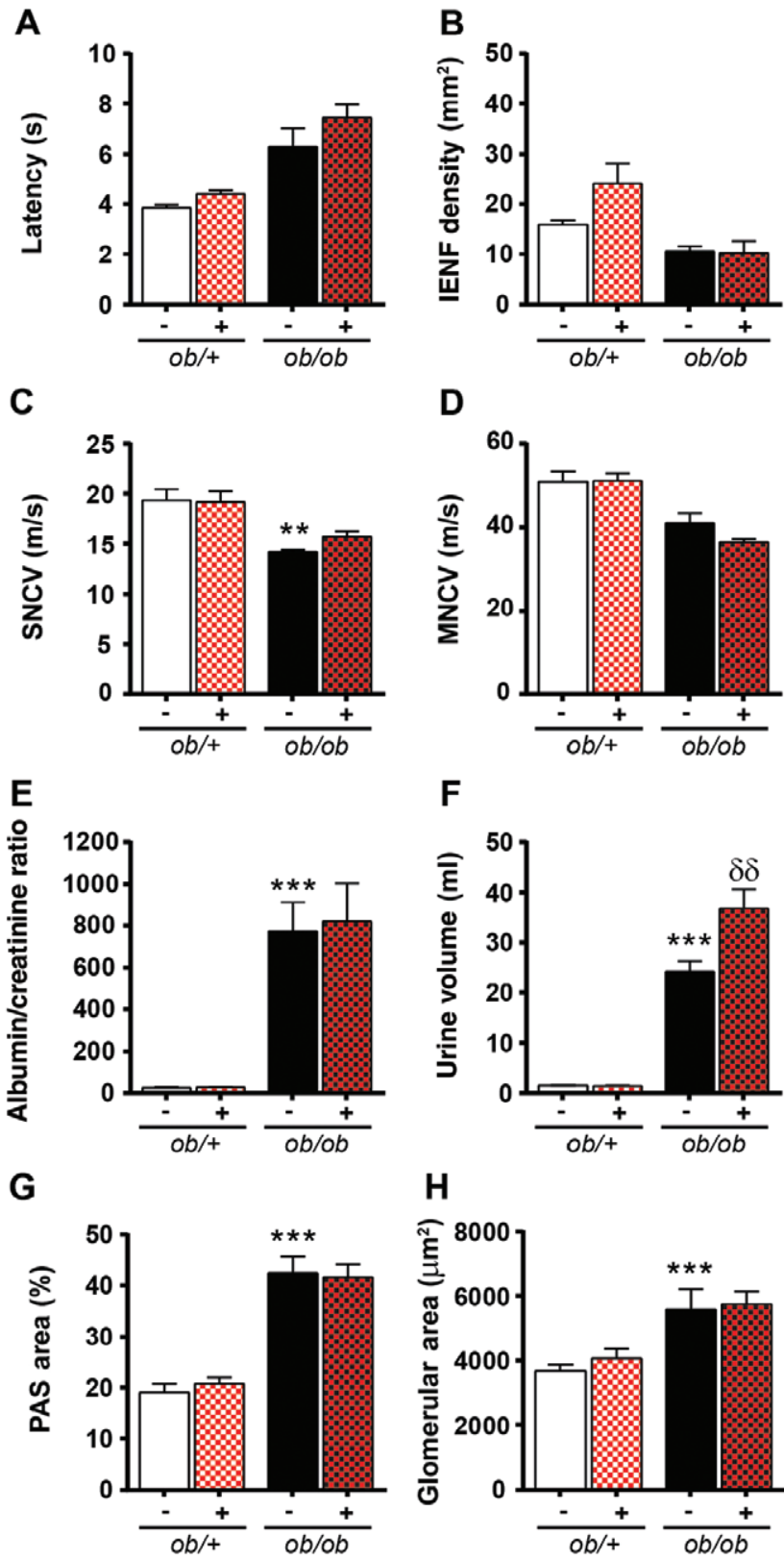

FIGURE 2 Effects of MK-0812 on DPN and DKD. Neuropathy phenotyping consisted of measuring latency of hindpaw withdrawal from a thermal stimulus (A), quantification of intraepidermal nerve fiber (IENF) density (B) and assessment of sural sensory (C) (SNCV) and sciatic motor (D) (MNCV) nerve conduction velocity.

Nephropathy was assessed by urinary ACR $(E)$, urine volume $(F)$, mesangial index, expressed as PAS-positive glomerular area divided by total glomerular area $(\mathrm{G})$ and total glomerular area $(\mathrm{H}) . * * P<.001$; $* * * P<.0001 \mathrm{ob} / \mathrm{ob}$ mice vs ob/+ mice. $\delta \delta P<.001 \mathrm{MK}-0812$-treated $o b /+$ and $o b / o b$ mice vs respective non-treated controls

\section{4 | DISCUSSION}

Recruitment of leukocytes to kidney tissue during T2D is an early event in the pathogenesis of DKD, ${ }^{10}$ and evidence suggests a similar mechanism in DPN. ${ }^{2}$ Previously, we reported that ob/ob mice develop an early and robust DPN, with transcriptomic bioinformatics analysis implicating "chemotaxis" and "inflammatory response" in DPN pathogenesis. ${ }^{13}$ As these animals manifest with early DKD, ${ }^{14}$ 
the current study sought to investigate whether targeting a common inflammatory pathway would have restorative effects by attenuating microvascular complications.

Contrary to our hypothesis, MK-0812 treatment evoked no improvement in DKD or DPN in ob/ob mice and was also insufficient in mitigating hyperglycaemia or hyperinsulinaemia. Despite the absence of predicted effects, however, MK-0812 decreased adipose inflammation in ob/ob mice by reducing concentrations of Cd68, F4/ 80 and Tnfo. ${ }^{13}$ MK-0812-treated ob/ob mice also displayed a modest decrease in \%GHb relative to untreated controls that could be attributed to the decrease in adipose tissue inflammation, but the improvement was insufficient to surmount the metabolic derangements characteristic of T2D. ${ }^{10}$ These results conflict with independent studies in which CCR2 inhibition prevented DKD in mice ${ }^{10}$ and humans. ${ }^{11}$ Kang et al. observed improvements in renal function of C57BKS $\mathrm{db}$ / $d b$ mice treated for 12 weeks with the CCR2 antagonist RS504393, ${ }^{10}$ while CCR2 inhibition reduced proteinuria in humans with T2D and DKD. ${ }^{11}$

We anticipate that the primary reason MK-0812 had largely no effect on metabolic parameters or diabetic complications is related to features of the ob/ob mouse model itself. Previous studies demonstrating a positive effect of CCR2 antagonists on DKD have almost exclusively used $d b / d b$ mice ${ }^{10}$ that do not develop the aggressive hyperglycaemia or DKD phenotype seen in ob/ob mice. ${ }^{14}$ This suggests that the likelihood of an intervention successfully attenuating disease in $d b / d b$ mice will be higher than that in a more aggressive T2D model. In addition, although $d b / d b$ mice lack the long isoforms of hypothalamic leptin receptor, short leptin receptor isoforms are expressed on the kidney and vasculature, and leptin-leptin receptor binding may confer a slight protection against DKD development that is absent in leptin-deficient ob/ob mice. ${ }^{15}$ Another caveat of this model stems from the fact that we observed no difference in markers of inflammation in plasma, kidney or nerve between control and ob/ ob mice. Inflammation is heavily implicated in DKD development in mouse models of T2D that have functional leptin. ${ }^{3}$ As leptin has numerous important immunomodulatory actions, a deficiency can compromise normal immune homeostasis. Hence, the atypical inflammatory signaling present in $o b / o b$ mice may not be suitable to explore therapies targeting inflammation. Observations from this study further suggest that DKD and DPN pathogenesis are independent of increases in kidney or nerve pro-inflammatory mediators in this model. Finally, we ensured that the lack of a positive effect on DKD/DPN was not the result of an ineffective dose of MK-0812 by looking directly at adipose tissue inflammation, a known driver of metabolic dysfunction. ${ }^{1}$ Similar to Kang et al. who demonstrated that CCR2 antagonism decreases adipose tissue inflammation in C57BKS $d b / d b$ mice, ${ }^{10}$ we observed a decrease in inflammatory markers following MK-0812 treatment in ob/ob mice, thus confirming that the lack of MK-0812 therapeutic efficacy on microvascular complications in ob/ob mice was not simply the result of inadequate drug activity.

In summary, MK-0812 had no effect on the metabolic parameters or the severity of DPN and DKD in ob/ob mice. These observations are unexpected, given previous independent reports of its efficacy in improving both glycaemic control and DKD in $d b / d b$ mice. ${ }^{10}$ We speculate that MK-0812 treatment in alternate models, such as $d b / d b$ or diet-induced obese mice, should be pursued, based on the existing literature supporting a role for inflammation in the onset and development of microvascular complications in humans as well as these other models.

\section{ACKNOWLEDGEMENTS}

The authors acknowledge the technical expertise of Ms. Jacqueline Dauch, Ms. Chelsea Lindblad, Ms. Jharna Saha and Dr. Sang Su Oh in conducting animal experiments. The authors thank the Hormone Core at Vanderbilt University and the Lipid Laboratory at the Mouse Metabolic Phenotyping Center at the University of Washington for mouse plasma measurements, respectively, and the Chemistry Core of the MDRTC (930DK020572) at the U-M for \%GHb measurements. The authors thank Dr. Charlie Alpers at the University of Washington for expert advice on the ob/ob mouse strain.

\section{Conflict of interest}

L. M. is employed as an Associate Principal Scientist at Merck Pharmaceuticals, Kenilworth, New Jersey. No other potential conflicts of interest relevant to this article were reported.

\section{Author contributions}

P. D. O. and L. M. H. directed the study, interpreted the data and wrote the manuscript. S. D. P. provided consultation, performed experimental work and contributed to the discussion of the manuscript. J. M. H, C. B. and H. Z. conducted animal experiments. L. M. was involved in study design, provided consultation and interpreted the pharmacokinetic data. S. A. S reviewed and edited the manuscript. E. L. F. and F. C. B. designed and directed the study, contributed to discussion and edited the manuscript. All authors critically revised the manuscript. E. L. F. is the guarantor of this work and, as such, had full access to all the data in the study and takes responsibility for the integrity of the data and accuracy of the data analysis.

\section{REFERENCES}

1. Hotamisligil GS. Inflammation and metabolic disorders. Nature. 2006;444:860-867.

2. Wilson NM, Wright DE. Inflammatory mediators in diabetic neuropathy. J Diabetes Metab. 2011;(suppl 5):004.

3. Navarro-Gonzalez JF, Mora-Fernandez C, Muros de Fuentes M, Garcia-Perez J. Inflammatory molecules and pathways in the pathogenesis of diabetic nephropathy. Nat Rev Nephrol. 2011;7:327-340.

4. Callaghan BC, Little AA, Feldman EL, Hughes RA. Enhanced glucose control for preventing and treating diabetic neuropathy. Cochrane Database Syst Rev. 2012 6:CD007543.

5. Nadkarni GN, Yacoub R, Coca SG. Update on glycemic control for the treatment of diabetic kidney disease. Curr Diab Rep. 2015;15:42.

6. Nguyen D, Ping F, Mu W, Hill P, Atkins RC, Chadban SJ. Macrophage accumulation in human progressive diabetic nephropathy. Nephrology (Carlton). 2006;11:226-231.

7. Herder C, Bongaerts BW, Rathmann W, et al. Differential association between biomarkers of subclinical inflammation and painful polyneuropathy: results from the KORA F4 study. Diabetes Care. 2015;38:91-96.

8. Doupis J, Lyons TE, Wu S, Gnardellis C, Dinh T, Veves A. Microvascular reactivity and inflammatory cytokines in painful and painless 
peripheral diabetic neuropathy. J Clin Endocrinol Metab. 2009;94:2157-2163.

9. Sullivan TJ, Miao Z, Zhao BN, et al. Experimental evidence for the use of CCR2 antagonists in the treatment of type 2 diabetes. Metabolism. 2013;62:1623-1632.

10. Kang YS, Lee MH, Song HK, et al. CCR2 antagonism improves insulin resistance, lipid metabolism, and diabetic nephropathy in type 2 diabetic mice. Kidney Int. 2010;78:883-894.

11. de Zeeuw D, Bekker P, Henkel E, et al. The effect of CCR2 inhibitor CCX140-B on residual albuminuria in patients with type 2 diabetes and nephropathy: a randomised trial. Lancet Diabetes Endocrinol. 2015;3:687-696.

12. Ruster $\mathrm{C}$, Wolf $\mathrm{G}$. The role of chemokines and chemokine receptors in diabetic nephropathy. Front Biosci. 2008;13:944-955.

13. O'Brien PD, Hur J, Hayes JM, Backus C, Sakowski SA, Feldman EL. BTBR ob/ob mice as a novel diabetic neuropathy model: neurological characterization and gene expression analyses. Neurobiol Dis. 2014;73:348-355.

14. Hudkins KL, Pichaiwong W, Wietecha T, et al. BTBR Ob/Ob mutant mice model progressive diabetic nephropathy. J Am Soc Nephrol. 2010;21:1533-1542.
15. Wang W, Poole B, Mitra A, et al. Role of leptin deficiency in early acute renal failure during endotoxemia in ob/ob mice. J Am Soc Nephrol. 2004;15:645-649.

\section{SUPPORTING INFORMATION}

Additional Supporting Information may be found online in the supporting information tab for this article.

How to cite this article: O'Brien PD, Hinder LM, Parlee SD, et al. Dual CCR2/CCR5 antagonist treatment attenuates adipose inflammation, but not microvascular complications in ob/ ob mice. Diabetes Obes Metab. 2017;19:1468-1472. https:// doi.org/10.1111/dom.12950 